\title{
Guidelines for the evaluation of instruments used in haematology laboratories*
}

\author{
NK SHINTON, JM ENGLAND, DA KENNEDY
}

Instruments have to undergo evaluation at three levels. Firstly, the manufacturer has to evaluate in order to produce data on which to base performance claims - for example, for inclusion in sales literature or for submission to a regulatory authority, such as the Food and Drug Administration in the USA. Secondly, evaluation is done on behalf of would-be users by laboratories acting for government departments and organisations such as the British Committee for Standardisation in Haematology and the College of American Pathologists. At this level, among other things, the evaluator seeks to validate the manufacturer's performance claims and look for inherent hazards in use. Thirdly, the would-be user evaluates whether a particular instrument-rather than the one tested elsewhere-is reliable and whether the special requirements of his own laboratory are met. In doing this he takes account of sample volume, workload and range of pathological states usually encountered.

The International Committee for Standardisation in Haematology (ICSH): "Protocol for type testing equipment and apparatus for haematological analysis" " provides general guidance for manufacturers. Shinton $e t a l^{2}$ have described a protocol for evaluation of coagulometers. Broughton et $a^{3}$ have recommended a scheme for the evaluation of instruments for use in clinical chemistry laboratories and Percy-Robb et al $^{4}$ have recommended a scheme for the evaluation of kits in the clinical chemistry laboratory.

The object of this paper is to provide general guidance and a systematic approach, to meet haematological requirements, for evaluation at the second and third levels. The principles outlined are common to the evaluation of a wide range of instruments and should be generally applicable to reagents and kits as well. The principles should enable the evaluator to adapt a protocol specifically for his own requirements. Appendix 2 is a protocol

\footnotetext{
*Prepared with the co-operation of the General Haematology Panel of the British Committee for Standardisation in Haematology.
}

Accepted for publication 8 March 1982 for evaluation of automated blood cell counters which illustrates the proposed approach. It is hoped that adoption of this format, which follows generally that of $\mathrm{ICSH}^{1}$ with some additions, will allow comparison of evaluation reports and exchange of information.

Evaluation at the second and third levels may be divided into a preliminary planning stage, followed by a technical stage which involves all the work necessary to assess the performance of the item under evaluation and to determine its practical use.

\section{Preliminary stage}

\section{1(a) General information}

Where relevant, the following should be checked before delivery: descriptive name of instrument, manufacturer and marketing company, list "price including that for optional attachments, rental or leasing arrangements, terms of guarantee, cost of maintenance contract, charges for visits, cost and availability of spare parts and consumables. It is helpful to know the length of time in use in the country of origin and approximate number and distribution, earlier models and/or antecedent apparatus.

\section{(b) Space and service requirements}

The following should be noted-floor area (if freestanding) or bench top space required, together with weight; details of the required electrical supply and other necessary services-for example, water (pressure and quality), compressed air, vacuum, drainage; need to provide a controlled environment-for example, for computerised equipment; any effect on the environment-for example, noise, viuration, heat production and any modification to the laboratory.

\section{(c) Instruction book}

This should be assessed in terms of clarity, lack of ambiguity and ease of reference. Particular attention should be made to that part which refers to maintenance carried out by the operator and "in-use" fault finding. 


\section{(d) Training}

If training is available; this should be undertaken before the evaluation starts. Assessment should be made of its effectiveness and of the facilities provided by the manufacturer-for example, manuals and training aids.

\section{(e) Methodology and principles or operation}

The following should be noted in the final report:

Instruments-Test(s) available; full specification, including principles of operation, degree of mechanisation or automation, data presentation and compatibility with other systems, alarm system for instrument failure; ancillary equipment needed-for example, mixing machine.

Consumables required (-for example, sample cups and reagents, controls and calibrators); description of packaging; storage requirements; formulation, if available, of reagents supplied or reccommended; as well as quantity supplied and number of tests/assays possible; ancillary consumables that are needed-for example, pipettes, tubes.

\section{(f) Planning of Technical Stage}

Plans should take account of length of time available to carry out the evaluation (specially important in the case of instruments which are borrowed or leased) and requirements for staff, reagents, reference materials and samples. Depending on the circumstances of the evaluation, a decision will have to be made whether to test only those claims made by the manufacturer or to investigate areas for which claims are not made. For example, a paediatric haematology laboratory may wish to test the effects of sample predilution on precision where the manufacturer has not considered such an application.

\section{SAMPLES}

Sufficient samples of test material-for example, whole blood, sera, should be available in high, low and intermediate levels of concentration or activity in a large enough volume to permit replicate testing. Division into aliquots and storage in a refrigerator or in the lyophilised state should be carried out where necessary. The type and concentration of anticoagulant must be considered as well as possible effects of predilution. Appropriate samples will be needed to study the effect of pathological changefor example, raised bilirubin, lipaemia, on the results.

3 REAGENTS (and consumables, where appropriate)

These should be those specified by the manufacturer or be their proven equivalent. Their storage and shelf life throughout the evaluation must be consi- dered. Sufficient must be available to complete testing from a single supply batch. NB-Some manufacturers may not wish to be responsible for the performance of an instrument unless their recommended reagents and/or consumables are used. At a later stage various batches of reagents should be assessed to determine whether or not batch variation exists.

\section{CONTROL AND CALIBRATION MATERIAL}

Sufficient volume must be obtained and the material should be stored appropriately throughout evaluation. Where calibration materials are supplied or recommended by the manufacturer the validity of assigned values needs consideration.

5 PARTICIPATION IN THE NATIONAL EXTERNAL QUALITY ASSESSMENT SCHEME (NEQAS)

Arrangements should be made with the Organiser for participating in NEQAS. The results will give an indication of the test instrument's performance as compared with others.

\section{RECORDS}

A detailed record should be kept. This should log all down-time, with causes; routine maintenance; significant events such as change of operator, batches of reagents or consumables; details of numbers of samples processed, in order to assess? throughput, and quantities of reagents, etc. used. It is also useful to record service engineers response time.

\section{Technical stage}

This involves all the work necessary to assess the performance of the items under evaluation and to calculate running and other costs.

Full evaluation requires that the following are considered:

1 Safety assessment, which should be undertaken first.

2 Scientific assessment of performance-that is, accuracy or compatibility with other results, effect of sample dilution (linearity), precision (within and between batch), carry-over.

3 Efficiency assessment including throughput, clinical usefulness, reliability, acceptability to user and costings.

\section{SAFETY ASSESSMENT}

\section{(a) Electrical}

Instruments should be checked by a competent authority for compliance with Electrical Safety Code for Hospital Laboratory Equipment. ${ }^{5}$ 


\section{(b) Mechanical}

Instruments should be examined for such things as moving parts, the presence of sharp edges and other hazards which might cause injury to the operator.

\section{(c) Microbiological}

Instruments should be tested for the production during normal operation of potentially infected droplets or aerosol. The most sensitive method requires the use of suspensions of bacterial spores, such as $B$ subtilis var globigii, and for this the services of an experienced microbiologist will be needed. However, in the case of serum or plasma samples an estimate of the degree of microbiological hazard can be obtained by putting fluorescent chemical markers, such as eosin or fluorescein, in the samples, placing clean, white absorbent paper over possible areas of contamination and then running the instrument. Droplet formation will be demonstrated by the presence of fluorescence under ultra-violet light. Comment should be made on the possibility of the user's hand becoming soiled with potentiallyinfected material; on the production of potentially infected effluent; and on the need to dispose of potentially infected consumables, such as reaction vessels, mixing devices and pipette tips. ${ }^{2}$

\section{(d) Chemical}

Consideration should be given to whether reagents are corrosive, carcinogenic, or toxic. The evaluation report should note whether appropriate warning notices are provided in literature and on packaging.

\section{(e) Radiation}

If the use of radioisotopes or a source of nonionising radiation is involved-for example, ultraviolet radiation or laser light, the advice of the Radiation Protection Officer should be sought and his comments incorporated into the report.

\section{SCIENTIFIC ASSESSMENT}

(a) Effect of dilution (linearity)

(i) Variables whose magnitude should be proportional to dilution It is desirable for an analytical method to give a linear relation over as large a range as possible and at least over the physiological and usually encountered pathological range. The linear relation should also pass through the origin.

Tests should be performed to give results at 10 concentrations evenly spaced-that is, 100,90 , $80 \ldots \ldots \ldots \ldots . . .20,10 \%$ values. Dilution of the test material is the usual method for obtaining the various concentrations. Diluting technique must be meticulous. It is recommended that when making dilutions only well calibrated, mechanical hand pipettes of accuracy and precision established by methods of $\mathrm{BS} 6018,{ }^{\circ}$ or pipettes complying with $\mathrm{BS} 1583^{7}$ or $\mathrm{BS} 1428^{8}$ should be used. The simplest method of analysing the results is to correct them for the dilution factor and see whether consistent answers are obtained over the relevant range of concentrations. More complex statistical analysis is given in Appendix 1.

(ii) Variables whose magnitude should be unaffected by dilution Certain haematological variables-for example, the red cell indices. should not be affected by dilution of the sample. Such data can be plotted out as the result against the concentration when a horizontal line with zero slope should be obtained. The method of statistical analysis is given in Appendix 1 .

\section{(b) Precision}

Precision is established by testing the sample on two or more occasions. It is usual to consider precision when the sample is assayed repeatedly within one batch of samples (within batch or replicate precision) as well as when the sample is assayed in two or more batches (between batch of duplicate precision). Precision may be quantified using the coefficient of variation (CV) as detailed in Appendix 1.

The organisation of the precision study will depend on the availability of samples. Where a sample is divided into aliquots each specimen container should be given a number and the order of sampling be regulated by use of a Table of random numbers. This procedure should minimise sampling errors. Ideally, precision studies should be made over the whole pathological range. Samples should certainly be examined in the high, low and intermediate ranges. When samples in any specified range are studied it is better to assay more samples fewer times than to assay a few samples more times but the final decision may have to be a compromise determined by availability of material. Sample storage and stability characteristics will determine whether it is possible to assay the sample in only two batches or whether they can be incorporated into several, so giving a better estimate of between batch variability. Blood counts on an automated system can only be done in relatively few batches-for example, two batches on the same day, the counter being switched off and recalibrated in between. Serum or plasma samples could be divided into aliquots, deep frozen and then assayed in many batches over a long period. The results should be analysed separately for each part of the pathological range using an analysis of variance, detailed of which are given in Appendix 1. Thus if samples are studied in the high, low, and 
intermediate ranges there will be three analyses of variance and three estimates of between and within batch precision. Exact details of number of samples per batch number of batches, etc will be given in documents describing specific evaluations.

\section{(c) Carry-over assessment}

This should be done according to the method of Broughton et $a l^{9}$ adapted to the specific circumstances of the evaluation if applicable. Details are given in Appendix 1.

\section{(d) Accuracy and comparability}

Accuracy is defined as "agreement between the best estimate of a quantity and its true value." 10 A true value may be considered to be one obtained by a definitive or reference method or that of a reference material. It is appropriate, therefore, to confine investigation of accuracy to areas where there are available definitive or reference methods or reference materials. Unfortunately at present there are no definitive methods and few reference materials in haematology.

Comparability may be defined as "the ability of the instrument ot produce results which agree satisfactorily with the results selected as the reference results." For example, in the case of a hospital laboratory the reference results are likely to be those obtained by the routine procedures.

A number of haematological measurements are made on instruments which have to be calibrated. Automated counters, for example, can only compare results on various samples and cannot assign values unless the instrument has been calibrated with suitable material. Instruments evaluation can be badly affected by this limitation since it can be difficult to decide whether a blood counter is working "incorrectly" or has been calibrated with a preserved blood whose assigned values are "incorrect". Thus when such instruments are compared (either with a reference method or with each other) it is more important to look at the general relation between the results-that is, the variation of the points about the regression line, rather than the actual slope of the regression line. For example, if Counter A always gave values $10 \%$ greater than Counter B this would be quite acceptable since there would be no variation of the points about the regression line and the "difference" could easily be eliminated by recalibration. It would be unacceptable, however, if Counter A gave answers 5\% higher on some samples and $50 \%$ higher on others; in this instance there would be serious variation about the regression line.

As many unselected samples should be studied as possible, the results analysed using the statistical procedure described in Appendix 1. If the unselected series does not give sufficient information about samples at the extremes of the pathological range, such samples should be selected and studied in detail separately. The full range of concentrations expected in clinical practice must be studied. The results obtained with the selected samples should not be merged with those in the unselected series since this would negate the statistical analysis.

\section{(e) Analysis of data}

General guidelines for the statistical analysis of results are given in Appendix 1. All the methods suggested are based upon the assumption that the observations are normally distributed and serious violations of this assumption may invalidate the statistical analysis. Measurements will not be normal if the frequency distribution curve is skewed or if the scatter of the results increase with magnitude-for example, when results by method $\mathrm{A}$ is plotted against those obtained by method $B$.

It may be that the distributions are normal when their logarithm is taken-for example, white cell and platelet counts and serum vitamin $B_{12}$; if so, the logarithms of the results should be analysed rather than the results themselves. In such complex situations further statistical advice may be necessay.

\section{EFFICIENCY ASSESSMENT}

An overall assessment should be made on the basis of the data available. Note should be made of any investigations that are outside the claims made for the instrument by the manufacturer.

\section{(a) Throughput}

This should be determined with reference to number of samples processed per batch with the inclusion of standards and drift control materials. The optimum number of samples that can be handled in a normal working day should be determined.

\section{(b) Clinical usefulness}

The suitability of the instrument for clinical diagnosis, screening and monitoring of therapy should be assessed. This may be subjective with a number of views possible.

\section{(c) Mechanical and electrical reliability}

These should be determined from reference to the written records made during evaluation.

\section{(d) Acceptability in practice}

Acceptability can be determined following discussion with the staff who operated the instrument. However, note should be taken of the level of experience of the user. 


\section{(e) Costings}

These should be prepared, taking account of reagent costs, consumable costs, capital and maintenance costs, and staff costs. Where appropriate, comparison may be made between the cost per test by the established method and the cost per test of the instrument under evaluation.

\section{Appendix I Statistical methods}

\section{A EFFECT OF DILUTION}

Variables whose magnitude should be proportional to dilution-for example, $\mathrm{Hb}$

One simple statistical method for assessing linearity is to compare how the data $(y)$ corresponds with parabolic and linear functions of the concentration (x) where:

$$
\begin{aligned}
& y=a_{1}+b_{1} x+c_{1} x^{2} \\
& y=a+b x
\end{aligned}
$$

If it can be shown that the parabolic relation is no better than the linear it is reasonable to assume the results are linear. A statistical test should then be performed to see whether the line passes through the origin-that is, does $a=0$ ?

\section{Example}

Results obtained by mixing packed cells from a patient with his own plasma. A concentration of $\mathbf{7 0 \%}$, for example, represents 70 parts cells to 30 parts plasma by volume. No results are given for zero concentration since no MCV can be obtained.

\begin{tabular}{lcl}
\hline Concentration (\%) & $H b(g / d l)$ & $M C V(f)$ \\
\hline 10 & $2 \cdot 0$ & 86 \\
20 & $4 \cdot 7$ & 86 \\
30 & $7 \cdot 5$ & 86 \\
40 & $10 \cdot 2$ & 87 \\
50 & 12.9 & 86 \\
60 & $15 \cdot 4$ & 87 \\
70 & $18 \cdot 0$ & 87 \\
80 & $20 \cdot 4$ & 86 \\
90 & 22.9 & 86 \\
100 & $25 \cdot 5$ & 87 \\
\hline
\end{tabular}

For simplicity of calculation the concentrations are considered to range from 1-10 rather than $10-100 \%$. The intermediate answers are rounded for the purposes of presentation and only if the exact values are carried through the calculation will the same final answer be produced.

From the concentrations $(x)$ and the haemoglobin results $(\mathrm{y})$ calculate number of observations $(\mathrm{n})$ and:

$$
\begin{array}{rll}
n=10 & \Sigma x=55 & \Sigma x^{2}=385 \\
& \Sigma x^{3}=3025 & \Sigma x^{4}=25333 \\
& \Sigma y=139 \cdot 5 & \Sigma y^{2}=2504 \cdot 77 \\
& \Sigma x y=981 \cdot 9 & \Sigma x^{2} y=7720 \cdot 9
\end{array}
$$

For parabolic fit calculate:

$$
\begin{aligned}
& I=\left(n \Sigma x^{3}\right)-\left(\Sigma x^{2} \Sigma x\right)=9075 \\
& J=\left(\Sigma x^{3} \Sigma x\right)-\left(\Sigma x^{2}\right)^{2}=18150 \\
& K=\left(\Sigma x^{2} \Sigma x y\right)-\left(\Sigma x^{3} \Sigma y\right)=-43956 \\
& L=\left(\Sigma x^{3} \Sigma x^{2}\right)-\left(\Sigma x^{4} \Sigma x\right)=-228690 \\
& M=\left(\Sigma x^{3}\right)^{2}-\left(\Sigma x^{4} \Sigma x^{2}\right)=-602580 \\
& N=\left(\Sigma x^{4} \Sigma x y\right)-\left(\Sigma x^{3} \Sigma x^{2} y\right)=1518750 \\
& a_{1}=(J N-K M) /(I M-J L)=-0.8183 \\
& b_{1}=-\left(K+a_{1} I\right) / J=2 \cdot 83098 \\
& c_{1}=-\left(n a_{1}+b_{1} \Sigma x-\Sigma y\right) / \Sigma x^{2}=-0.020833
\end{aligned}
$$

For linear fit calculate:

$\mathrm{b}=(\Sigma x y-(\Sigma x \Sigma y / n)) /\left(\Sigma x^{2}-(\Sigma x \Sigma x / n)\right)=2 \cdot 601818$

$\mathrm{a}=(\dot{\Sigma} \mathrm{y} / \mathrm{n})-(\mathrm{b} \Sigma \mathrm{x} / \mathrm{n})=-0.36$

To compare parabolic with linear fit calculate:

$$
\begin{array}{r}
F=(n-3)\left[\frac{\Sigma y^{2}-a \Sigma y-b \Sigma x y}{\Sigma y^{2}-a_{1} \Sigma y-b_{1} \Sigma x y-c_{1} \Sigma x^{2} y}-1\right] \\
=45.16
\end{array}
$$

Refer to tables of $F$ with 1 and ( $n-3)$ degrees of freedom-that is, in this example 1 and 7 degrees of freedom for which $F=5.59$ when $p=0.05$ Since the value of $F$ found exceeds 5.59 the parabolic fit is better than the linear - that is, the results are not linear. If the result for $F$ had been $<5.59$ there would have been no reason to suspect a departure from linearity and it would have been reasonable to see whether " $a$ " differed from zero as described in section D.

Variables whose magnitude should be unaffected by dilution-for example, $M C V$

The data should be analysed as above to see whether a parabolic relation is better than a linear one. If the data appears to be linear the slope of the line should be tested to see if it is zero-that is, does $b=0$ ? (See section $\mathrm{D}$ for calculation). The variable can be assumed to be unaffected by dilution if the parabolic relation is no better than the linear one and " $b$ " is not significantly different from zero.

Example From the concentrations (x) divided by 10 and the MCV results (y) given above calculate:

$$
\begin{aligned}
& \mathrm{n}=10 \quad \Sigma \mathrm{x}=55 \quad \Sigma \mathrm{x}^{2}=385 \\
& \Sigma x^{3}=3025 \quad \Sigma x^{4}=25333 \\
& \Sigma y=864 \quad \Sigma y^{2}=74652 \\
& \Sigma x y=4757 \quad \Sigma x^{2} y=33311 \\
& \begin{array}{lll}
I=9075 & J=18150 & K=-782155 \\
L=-228690 & M=-602580 & N=1974330 \\
a_{1}=85.73 & b_{1}=0.227273 & c_{1}=-0.0151515 \\
b=0.060606 & a=86.07 & F=0.43
\end{array}
\end{aligned}
$$

Since $F<5.59$ there is no reason to suspect a 
departure from linearity and a test should now be made to see if $b=0$ (see section $D$ for method)

\section{B BETWEEN AND WITHIN BATCH PRECISION}

An analysis of variance is performed for each group of samples in a specific part of the pathological range-for example, several samples in the intermediate range are assayed repeatedly in several batches. For simplicity an example will be given of red cell counts on four samples analysed in triplicate in two batches. In practice a larger experiment would be performed.

Example The data should be presented as shown below and the sub-totals, column totals, grand-total, and mean calculated.

\begin{tabular}{|c|c|c|c|c|}
\hline & \multicolumn{2}{|l|}{ Batch I } & \multicolumn{2}{|l|}{ Batch 2} \\
\hline & Observation & Subtotal & Observation & Subtotal \\
\hline Sample 1 & $\left.\begin{array}{l}4 \cdot 64 \\
4 \cdot 66 \\
4 \cdot 72 \\
4 \cdot 17\end{array}\right\}$ & $14 \cdot 02$ & $\left.\begin{array}{l}4 \cdot 85 \\
4 \cdot 91 \\
4 \cdot 87 \\
4 \cdot 28\end{array}\right\}$ & $14 \cdot 63$ \\
\hline Sample 2 & $\left.\begin{array}{l}4 \cdot 17 \\
4 \cdot 17 \\
5 \cdot 17\end{array}\right\}$ & $12 \cdot 51$ & $\left.\begin{array}{l}4 \cdot 25 \\
4 \cdot 28 \\
5 \cdot 13\end{array}\right\}$ & $12 \cdot 81$ \\
\hline Sample 3 & $\left.\begin{array}{l}5 \cdot 20 \\
5 \cdot 18 \\
5 \cdot 43\end{array}\right\}$ & $15 \cdot 55$ & $\left.\begin{array}{l}5 \cdot 35 \\
5 \cdot 24 \\
5 \cdot 51\end{array}\right\}$ & $15 \cdot 90$ \\
\hline Sample 4 & $\left.\begin{array}{l}5 \cdot 39 \\
5 \cdot 44\end{array}\right\}$ & $16 \cdot 26$ & $\left.\begin{array}{l}5.47 \\
5.51\end{array}\right\}$ & 16.49 \\
\hline $\begin{array}{c}\text { Column } \\
\text { total }\end{array}$ & & $58 \cdot 34$ & & 59.83 \\
\hline
\end{tabular}

Grand total $=58 \cdot 34+59 \cdot 83=118 \cdot 17$.

Mean $=118 \cdot 17 / 24=4 \cdot 92375$.

If the number of samples, batches and replicates are $\mathrm{u}, \mathrm{v}$ and $\mathrm{n}$ respectively then the sums of squares (SS) are:

Between batch $\mathrm{SS}=\left(\Sigma(\text { Column total })^{2} / \mathrm{un}\right)-$ ((grand total) $)^{2} /$ uvn)

$=\left(\left(58.34^{2}+59.83^{2}\right) / 4 \times 3\right)-\left(118.17^{2} / 4 \times 2 \times 3\right)$

$=0.09250$

Between replicate $S S=$ ( $\Sigma$ (individual observation $\left.)^{2}\right)-\left(\sum(\text { sub-total })^{2} / \mathrm{n}\right)$

$=\left(4 \cdot 64^{2}+4 \cdot 66^{2} \ldots .5 \cdot 47^{2}+5 \cdot 51^{2}\right)-\left(\left(14 \cdot 02^{2}+\right.\right.$

$\left.\left.14 \cdot 63^{2} \ldots 16 \cdot 26^{2}+16 \cdot 49^{2}\right) / 3\right)$

$=0.015067$

The SSs may then be converted to CVs as follows:

Between batch $\mathrm{CV}=$

$\sqrt{\frac{(\text { between batch SS }) /(\mathrm{v}-1)}{\text { mean }}} \times 100 \%=6.177 \%$

Between replicate $\mathrm{CV}$ within batch $=$

$\sqrt{\frac{\text { (between replicate SS }) /(\mathrm{uv}(\mathrm{n}-1))}{\text { mean }}} \times 100 \%=0.623 \%$
C CARRY-OVER ASSESSMENT

Test a high sample consecutively thrice $\left(i_{1}, i_{2}, i_{3}\right)$ followed immediately by testing a low sample consecutively thrice $\left(\mathrm{j}_{1}, \mathrm{j}_{2}, \mathrm{j}_{3}\right)$. Carry-over \% is then:

$$
\frac{\left(j_{1}-j_{3}\right)}{\left(i_{3}-j_{3}\right)} \times 100 \%
$$

Example Results obtained for red cell counts were $6 \cdot 28,6 \cdot 27,6 \cdot 30,0.74,0.52,0.49$

Carry-over $=\frac{(0.74-0.49)}{(6.30-0.49)} \times 100=4.30 \%$

D LINEAR REGRESSION ANALYSIS

Regression analysis may be used to study the effect of dilution by plotting result (y) against concentration $(x)$ and fitting a line

$$
y=a+b x
$$

as described in section $\mathrm{A}$. In this case concentration $(x)$ is assumed to be error free and only one a and bo coefficient can be calculated, those for the regres sion line of $y$ and $x$-that is, the line to be used if given $\mathrm{x}$, one wishes to calculate $\mathrm{y}$. It is, therefore; quite appropriate to test whether $a$ or $b=0$.

However, regression analysis can also be used to assess accuracy or comparability by plotting the result $(y)$ by the new method against $(x)$ the one by the reference or best available method. In this case there are two regression lines, for $\mathrm{y}$ on $\mathrm{x}$ and for $\mathrm{x}$ on $y$, depending on whether $y$ is to be predicted from $x$ or vice versa.

Because there are two regression lines there are two possible values for $a$ and two for $b$. If the two values for $b$ are multiplied together and the square root taken the correlation coefficient, $r$, is obtained (note that $r$ should not be calculated for the effect of dilution since there is only one regression line). The correlation coefficient is usually very close to 1.0 when methods are compared and is therefore of very little use for assessing how well methods agree. Instead the $\mathrm{SD}$ of the points about the regression line (of $\mathrm{y}$ on $\mathrm{x}$ ) should be determined.

Example Packed cell volumes (PCV) on 18 samples were determined on the automated counter being tested (y values) and the best available automated counter ( $x$ values). The data have been multiplied by 1000 for convenience and are presented below. In practice considerably more than 18 samples would be studied. 


\begin{tabular}{llllll}
\hline$x$ & $y$ & $x$ & $y$ & $x$ & $y$ \\
\hline 281 & 249 & 422 & 361 & 489 & 383 \\
291 & 235 & 453 & 393 & 510 & 433 \\
338 & 287 & 463 & 394 & 523 & 453 \\
365 & 284 & 464 & 386 & 531 & 452 \\
385 & 321 & 464 & 387 & 618 & 507 \\
417 & 345 & 472 & 393 & 681 & 570 \\
\hline
\end{tabular}

Calculate the following:

$$
\begin{array}{lll}
n=18 & \Sigma x=8167 & \Sigma x^{2}=3884659 \\
& \Sigma y=6833 & \Sigma y^{2}=2721177 \\
& \Sigma x y=3249975
\end{array}
$$

For the regression line of $y$ on $x$ :

$\mathrm{b}=\frac{\Sigma x y-\left(\Sigma x \sum y / n\right)}{\Sigma x^{2}-\left((\Sigma x)^{2} / n\right)}=0.83575$

$\mathrm{a}=(\Sigma \mathrm{y} / \mathrm{n})-(\mathrm{b} \Sigma \mathrm{x} / \mathrm{n})=0.41226$

The scatter of the points about this regression line has a variance

$$
s^{2}=\frac{\Sigma y^{2}-\frac{(\Sigma y)^{2}}{n}-\left(b^{2} \Sigma x^{2}\right)+\frac{(b \Sigma x)^{2}}{n}}{n-2}=136.875
$$

and a standard deviation

$\mathrm{s}=\sqrt{136 \cdot 875}=11.699$

The correlation coefficient

$$
\mathrm{r}=\mathrm{b} \sqrt{\frac{\mathrm{n} \Sigma \mathrm{x}^{2}-(\Sigma \mathrm{x})^{2}}{\mathrm{n} \mathrm{y}^{2}-(\Sigma \mathrm{y})^{2}}}=0.99136
$$

To determine whether $\mathbf{a}=0$

calculate:

$t=\frac{\mathrm{a}}{\mathrm{s}} \sqrt{\frac{\mathrm{n \Sigma \textrm {x } ^ { 2 } - ( \Sigma \mathrm { x } ) ^ { 2 }}}{\Sigma \mathrm{x}^{2}}}=0.0321$

and refer the value of $t$ to Tables of Student's $t$ in which $t=2.12$ when $\mathrm{p}=0.05$ and there are $\mathrm{n}-2=$ 16 degrees of freedom. Since the calculated value of $t<2 \cdot 12$ there is no reason to believe that " $\mathrm{a}$ " differs from zero.

To determine whether $\mathrm{b}=0$

calculate:-

$t=\frac{\mathrm{b}}{\mathrm{s}} \sqrt{\Sigma \mathrm{x}^{2}-\frac{(\Sigma \mathrm{x})^{2}}{\mathrm{n}}}=30 \cdot 233$

and refer to Tables of Student's $t$ with $\mathrm{n}-2=16$ degrees of freedom. Since $t>2 \cdot 12$, it is reasonable to conclude that " $b$ " differs from zero.

\section{Appendix II Model protocol for the evaluation of automatic blood cell counters}

This protocol provides a standard format of evaluation for use by clinical laboratories wishing to assess an automatic blood cell counter.
PLANNING

Instrument calibration

The method of calibration will have to be determined for each specific instrument due to differences in mode of operation.

\section{Samples}

Fresh blood samples, collected through a needle of bore 19G, obtained from volunteer donors and patients who have given consent following explanation of the purpose to which the blood will be used. These will be required from subjects with the following states:
A Healthy donor
B Healthy donor with lipaemia (post-prandial)
C Polycythaemia-Hb $18-20 \mathrm{~g} / \mathrm{dl}$
D Microcytic anaemia-MCV $<75 \mathrm{fl}$, haemog- lobin $10-11 \mathrm{~g} / \mathrm{dl}$
E Leucocytosis-WBC $20-40 \times 10^{9} / 1$
F Leucopenia-WBC 0.5-2.0 $\times 10^{9} / 1$
G Thrombocytosis—platelets 500-700 $\times 10^{9} / 1$
$\mathrm{H}$ Thrombocytopenia-platelets 50-80 $\times 10^{\%} / \mathrm{l}$
I Macrocytic anaemia-MCV $>100 \mathrm{fl}$, haemoglobin $10-11 \mathrm{~g} / \mathrm{dl}$
J Jaundice-serum bilirubin $>30 \mu \mathrm{mol} / \mathrm{l}$
K Myelomatosis-paraprotein detected by immunoelectrophoresis

The volume of blood to be collected will depend upon the requirements of the instrument under test. A calculation for each part of the assessment must be made. In view of the total volume of blood required, it will probably be necessary to carry out each part of assessment on samples from different subjects. For the same reason, precision of haemoglobin and red cell counting at the lower end of the range must be based on at least 10 paired samples.

\section{Reference material}

ICSH-related hemiglobincyanide (HiCN) reference preparation ${ }^{1}-10 \mathrm{ml}$

Red cell, white cell, and platelet reference material (if available) $-10 \mathrm{ml}$

\section{External quality assessment samples}

Arrangements should be made with the organiser of the UK National external quality assessment scheme, and if appropriate the Regional quality assessment scheme, for supply of material during the trial period.

\section{ASSESSMENT OF PERFORMANCE}

I Effect of dilution (linearity)

Tests should be performed to give results at concentrations evenly spaced within the limitation of 
the specific instrument for the following:

(i) Haemoglobin and red cell count using a polycythaemic sample diluted in patient's own plasma. Linearity of $\mathrm{MCV}, \mathrm{MCH}$ and $\mathrm{MCHC}$ can be performed from this experiment.

(ii) White cell count using leucocytosis sample diluted in patients own plasma.

(iii) Platelet count using thrombocythaemic sample diluted in patients own plasma.

\section{Precision}

Replicate testing of whole blood samples A-K

(i) within 2 hours of collection.

(ii) 4-6 hours later with instrument switched off after completion of experiment and then recalibrated.

(iii) 24 hours following collection, after storage at $4^{\circ} \mathrm{C}$.

III Carry-over

(i) Test blood $\mathrm{C}$ three times followed immediately

by blood $\mathrm{D}$ three times.

(ii) Test blood $\mathrm{E}$ three times followed immediately by blood $\mathrm{F}$ three times.

(iii) Test blood $\mathrm{G}$ three times followed immediately by blood $\mathrm{H}$ three times.

\section{Accuracy and comparability}

(i) Haemoglobin Test samples from high, intermediate, and low range, the haemoglobin concentrations of which have been determined by the ICSH reference method ${ }^{12}$ (calibrated by $\mathrm{HiCN}$ ).

(ii) Packed red cell volume Test samples for high, intermediate and low range whose PCV has been determined by the ICSH reference method. ${ }^{13}$

(iii) Cell counting Compare test instrument results obtained on 10 samples from each of the conditions listed above with those obtained by the reference method on the same samples.

(iv) Where the instrument has a separate channel for sampling prediluted specimens-for example, capillary blood from children, a comparison should be made with results from whole blood of the same subject.

(v) Throughout Determine maximum through-put.

\section{$V$ Clinical Usefulness}

With reference to the results of technical evaluation and determination of throughput, the place of the instrument in a routine haematological department should be stated-that is, its suitablility for clinical diagnosis, screening and monitoring of therapy.

\section{Acceptability in practice}

Comments should be made on staff reaction and maintenance problems. Number of "down-times" and reasons for these should be documented together with time before machine was again operational.

Members of the General Haematology Panel

NK SHINTON Coventry and Warwickshire Hospital

P CROSLAND-TAYLOR Middlesex Hospital, London

DW DAWSON North Manchester General Hospital

JM ENGLAND Watford General Hospital

DA KENNEDY Scientific and Technical Research, Supply Division, DHSS

SM LEWIS Royal Postgraduate Medical School

RM ROWAN Western Infirmary, Glasgow

\section{References}

'International Committee for Standards in Haematology: Protocol for type testing equipment and apparatus used for haematological analysis. J Clin Pathol 1978;31:275-9.

' Shinton NK, Bloom AL, Colvin BT, Flute PT, Preston FE, Kennedy DA. Tentative protocol for the evaluation of coagulometers based on one-stage prothrombin time. Clin Lab Haematol 1981;3:71-6.

${ }^{3}$ Broughton PMG, Gowenlock AH, McCormach JJ, Neill DW. A revised scheme for the evaluation of automated instruments for use in clinical chemistry. Ann Clin Biochem 1974;11: 207-18.

${ }^{4}$ Percy-Robb IW, Broughton PMG, Jennings RD, et al.A recommended scheme for the evaluation of kits in the clinical chemistry laboratory. Ann Clin Biochem 1980;17:217-26.

${ }^{5}$ Electrical Safety Code for Hospital Laboratory Equipmentce DHSS, Scottish Home and Health Department, Department of Health and Social Services, Northern Ireland and Welsh Office, HMSO: 1977.

${ }^{\circ}$ British Standard 1583: One mark pipettes London: British Standards Institution, 1961.

${ }^{7}$ British Standards 1428: Part D4: Capillary pipettes London: British Standards Institution, 1963.

${ }^{8}$ British Standards 6018: Method of test for accuracy and preciston of hand pipettes London: British Standards Institution, 1981.

${ }^{9}$ Broughton PMG, Buttolph MA, Gowenlock AH, Neill DW, Skentlebury RG. A recommended scheme for the evaluation of instruments for automated analysis in the clinical biochemistry laboratory. J Clin Pathol 1969;22:278-84.

${ }^{10}$ International Federation of Clinical Chemistry, Expert Group on Diagnostic Kits and Reagents (Chairman M Rubin). Provisional Recommendation on Evaluation of Diagnostic Kits Part 2 Guidelines for the Evaluation of Clinical Chemistry Kits. Clin Chim Acta 1978;95:163F-8F.

"British Standard 3985: Specification for cyanmethaemoglobin (hemiglobincyanide) solution for photometric haemog. lobinometry. London: British Standards Institution, 1978.

12 International Committee for Standardisation in Haematology. Recommendations for reference method for haemoglobinometry in human blood (ICSH Standard EP 6/2: 1977) and specifications for international haemiglobincyanide reference preparation (ICSH Standard EP 6/3: 1977). J Clin Pathol 1978;31:139-43.

${ }^{13}$ International Committee for Standardisation in Haematology Expert Panel on Blood Cell Sizing. Recommendation for reference method for determination by centrifugation of packed cell volume of blood. J Clin Pathol 1980;33:1-2.

Requests for reprints to: Dr NK Shinton, Coventry and Warwickshire Hospital, Stoney Stanton Road, Coventry CV1 4FH, England. 